\title{
Assessment of extrahepatic abdominal extension in primary malignant liver tumours of childhood
}

\author{
Derek J. Roebuck • Neil J. Sebire • Danièle Pariente
}

Received: 28 March 2007 / Accepted: 20 April 2007 / Published online: 26 May 2007

(C) Springer-Verlag 2007

\begin{abstract}
Imaging is a crucial part of the evaluation of children with primary liver malignancies. In addition to staging the intrahepatic extent of the tumour, the 2005 revision of the PRETEXT system specifies certain criteria for assessment of extrahepatic disease. This article reviews the imaging findings of two of these criteria: direct extrahepatic extension of the tumour (E1, E1a, E2, E2a) and tumour rupture at diagnosis $(\mathrm{H} 1)$.
\end{abstract}

Keywords Hepatoblastoma $\cdot$ Hepatocellular carcinoma .

Staging $\cdot$ Metastasis

\section{Introduction}

There are two main staging systems for primary malignant liver tumours of childhood. The system used by the Children's Oncology Group, predominantly in North America, is based mainly on the surgical findings and extent of resection of the tumour, and is most appropriate for use in treatment protocols that favour immediate surgery at diagnosis $[1,2]$. In contrast, the PRETEXT system is

\section{J. Roebuck ( $\bowtie)$}

Department of Radiology, Great Ormond Street Hospital,

London WC1N 3JH, UK

e-mail: roebud@gosh.nhs.uk

\section{N. J. Sebire}

Department of Anatomical Pathology,

Great Ormond Street Hospital,

London, UK

D. Pariente

Centre Hospitalo-Universitaire de Bicêtre APHP,

Le Kremlin-Bicêtre, France designed to evaluate tumours regardless of whether primary surgery is performed, and is used by the International Childhood Liver Tumor Strategy Group (SIOPEL) [3]. With increasing experience and international cooperation it has become clear that each system has its own merits, and the two are not mutually exclusive. The PRETEXT system is likely to be used for the purpose of making comparisons between trials conducted by different groups.

Various criteria for assessment of vascular invasion and extrahepatic spread of tumour are important parts of the 2005 revision of the PRETEXT system [3]. Different forms of extrahepatic tumour spread are distinguished, according to whether they involve direct extension or peritoneal disease (E1, E1a, E2, E2a), tumour rupture at diagnosis (H1), lymph node metastasis (N1, N2), or other forms of metastasis (M1).

Although the PRETEXT system can be used to stage any primary liver tumour, it is most often used in hepatoblastoma, which is the most common primary malignant liver tumour of childhood, even in areas where the prevalence of hepatitis B is high $[4,5]$. In the current SIOPEL system, the presence of any of the criteria E1, E1a, E2, E2a, H1, M1, $\mathrm{N} 1$ or $\mathrm{N} 2$ in a child with hepatoblastoma mandates treatment on a high-risk protocol. Although the new Children's Oncology Group protocol for treatment of hepatoblastoma is not finalized, it will probably also rely to a large extent on imaging for risk stratification. The identification of these forms of spread is therefore crucial to accurate patient management.

This article reviews the imaging findings of direct tumour extension and peritoneal disease (E1, E1a, E2, E2a) and tumour rupture at diagnosis (H1). The technical aspects of imaging liver tumours in children have recently been reviewed by Roebuck et al. [6]. 

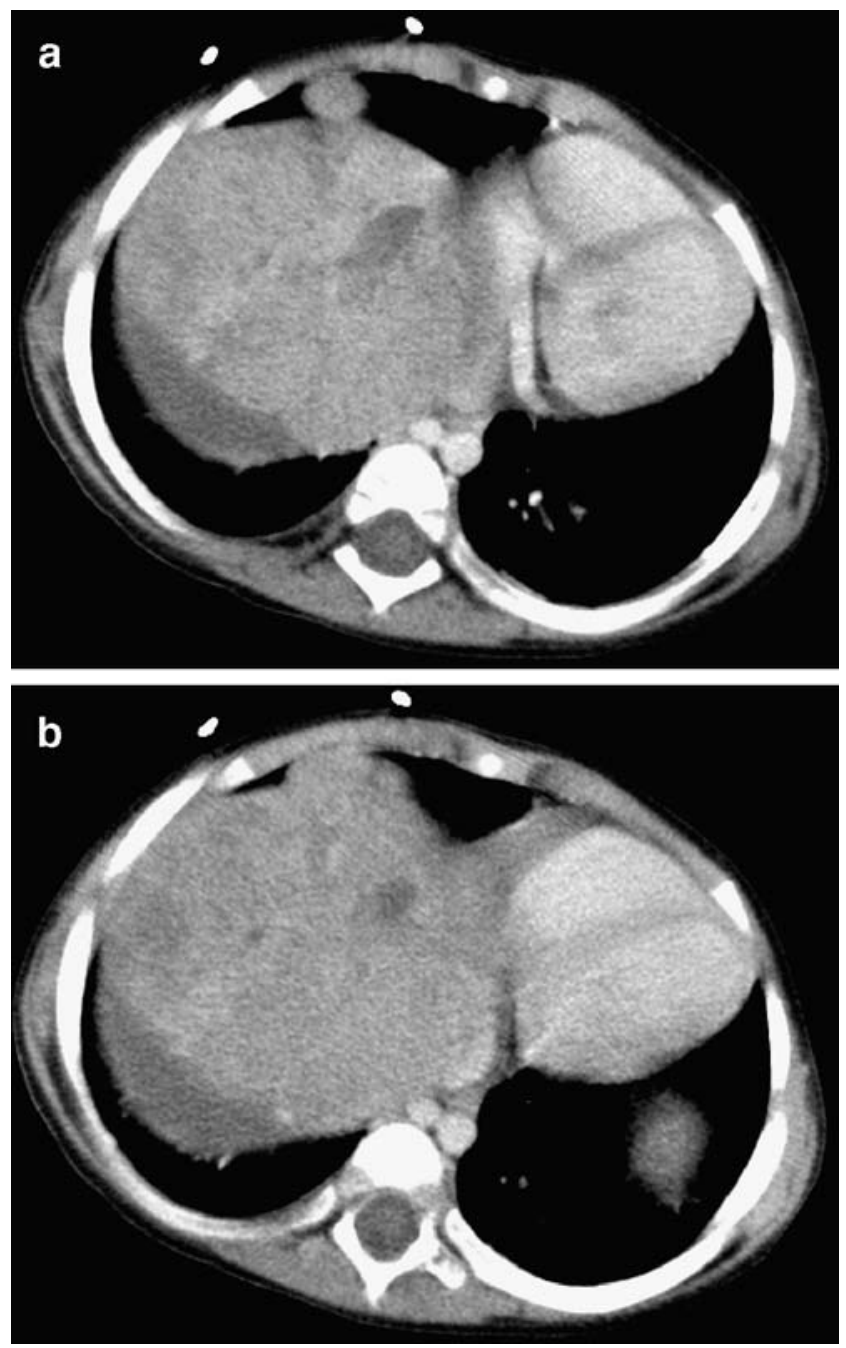

Fig. 1 Extension of hepatoblastoma through the diaphragm (E1). Contrast-enhanced CT at two levels shows growth of the primary tumour through the diaphragm into the thorax

\section{Extrahepatic abdominal disease (E1, E1a, E2, E2a)}

In the SIOPEL 3 study, 17 (4.1\%) of 417 patients with hepatoblastoma registered before the end of 2004 were classified as E+ (M. Childs, personal communication). The original PRETEXT system, which was used in this study, included patients with lymph node metastases in this group. This indicates that direct extension of tumour, as detected by imaging findings at diagnosis, is clearly very uncommon in hepatoblastoma. Isolated extrahepatic spread is even less common: only 6 of the 17 patients $(1.4 \%$ of those with hepatoblastoma) were classified as high risk on this basis alone. As a consequence, until the 2005 revision of the PRETEXT system, all SIOPEL protocols required that extrahepatic abdominal spread of tumour $(\mathrm{E}+)$, including lymph node metastases, be proved by biopsy. It was unfortunate that this rule failed to distinguish between different tumour types. A second problem was that some types of direct tumour spread (through the diaphragm, for example) are difficult to biopsy safely. In addition, the requirement for biopsy proof prevented the full use of the emerging capabilities of modern imaging techniques.

In the revised system, two stages of intra-abdominal spread are recognized. In the first, E1, there is direct growth of tumour into adjacent structures. This must be distinguished from an exophytic tumour growth pattern. In fact, hepatoblastoma often grows in a pedunculated manner, and when there is no unequivocal evidence of invasion of adjacent structures, tumours of this type are regarded as being confined to the liver [3].

In principle, the tumour may grow into any of the structures that are anatomically related to the liver.
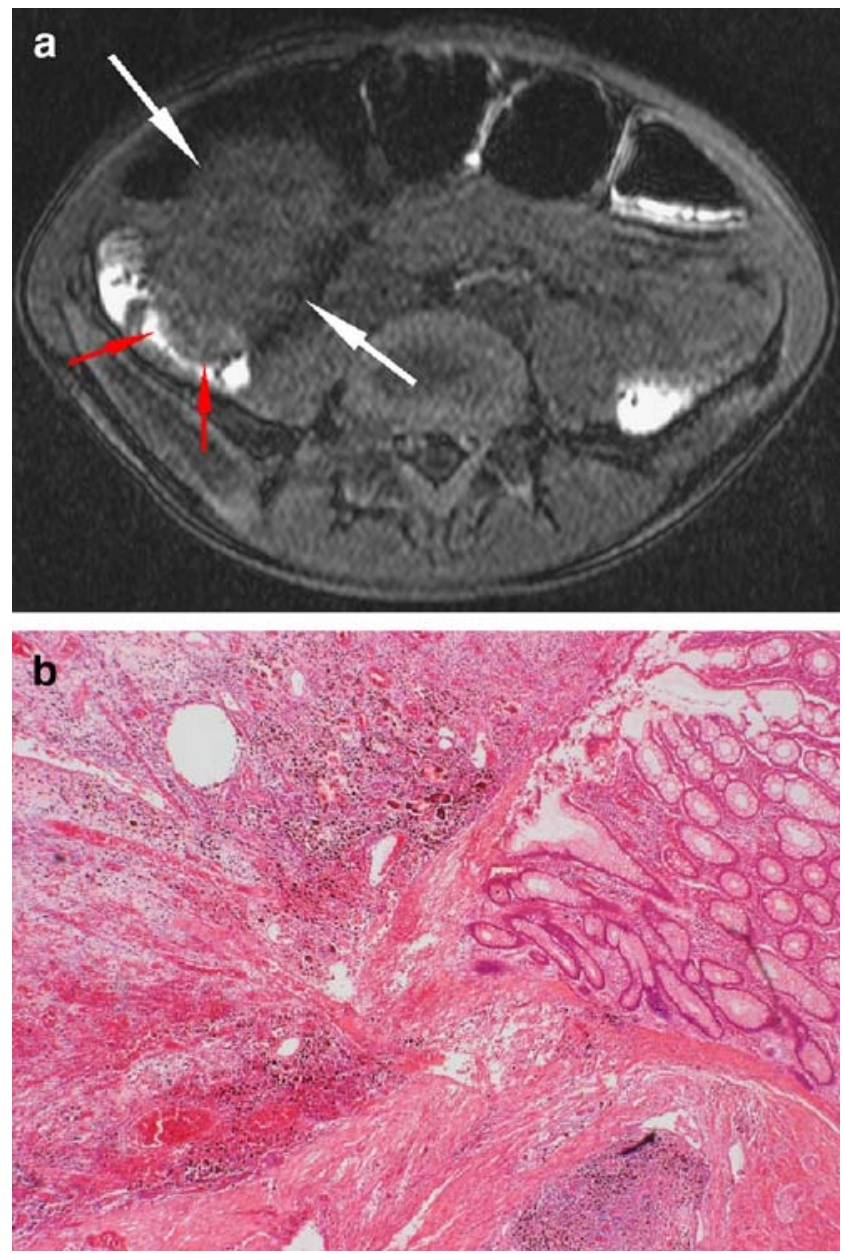

Fig. 2 Extension of hepatoblastoma into the hepatic flexure of the colon (E1). a Transverse MR image shows extension of a tumour arising in segment 6 of the liver (white arrows) into the colon (red arrows). b At surgery, the hepatic flexure was densely adherent to the liver, and the right lobe and this section of bowel were resected en bloc. Histological sections revealed direct invasion of the bowel wall. The photomicrograph demonstrates colonic mucosa on the right with underlying submucosal tumour showing marked chemotherapy-associated change. There is puckering of the muscularis mucosae, indicating previous involvement by viable locally invasive tumour at this point $(\mathrm{H} \& \mathrm{E}$, original magnification $\mathrm{x} 40)$. The patient had suffered episodes of gastrointestinal bleeding 
Although it is uncommon in hepatoblastoma, about $10 \%$ of adults with hepatocellular carcinoma have diaphragmatic adhesion or invasion by tumour [7]. At the time the original PRETEXT system was devised, there was no good preoperative method for assessing involvement of the diaphragm [7]. Diaphragmatic penetration by tumour may now be shown convincingly by MRI or CT (Fig. 1). Coronal or sagittal images may be more helpful than transverse images in this context. Subtle diaphragmatic invasion and tumour adhesion to the diaphragm without tumour invasion will be missed at cross-sectional imaging.

Invasion of the duodenum or colon (Fig. 2) appears to be quite rare in both hepatoblastoma and hepatocellular carcinoma, although there is little published information on this subject. There may also be direct extension of tumour into the retroperitoneum, with involvement of the pancreas
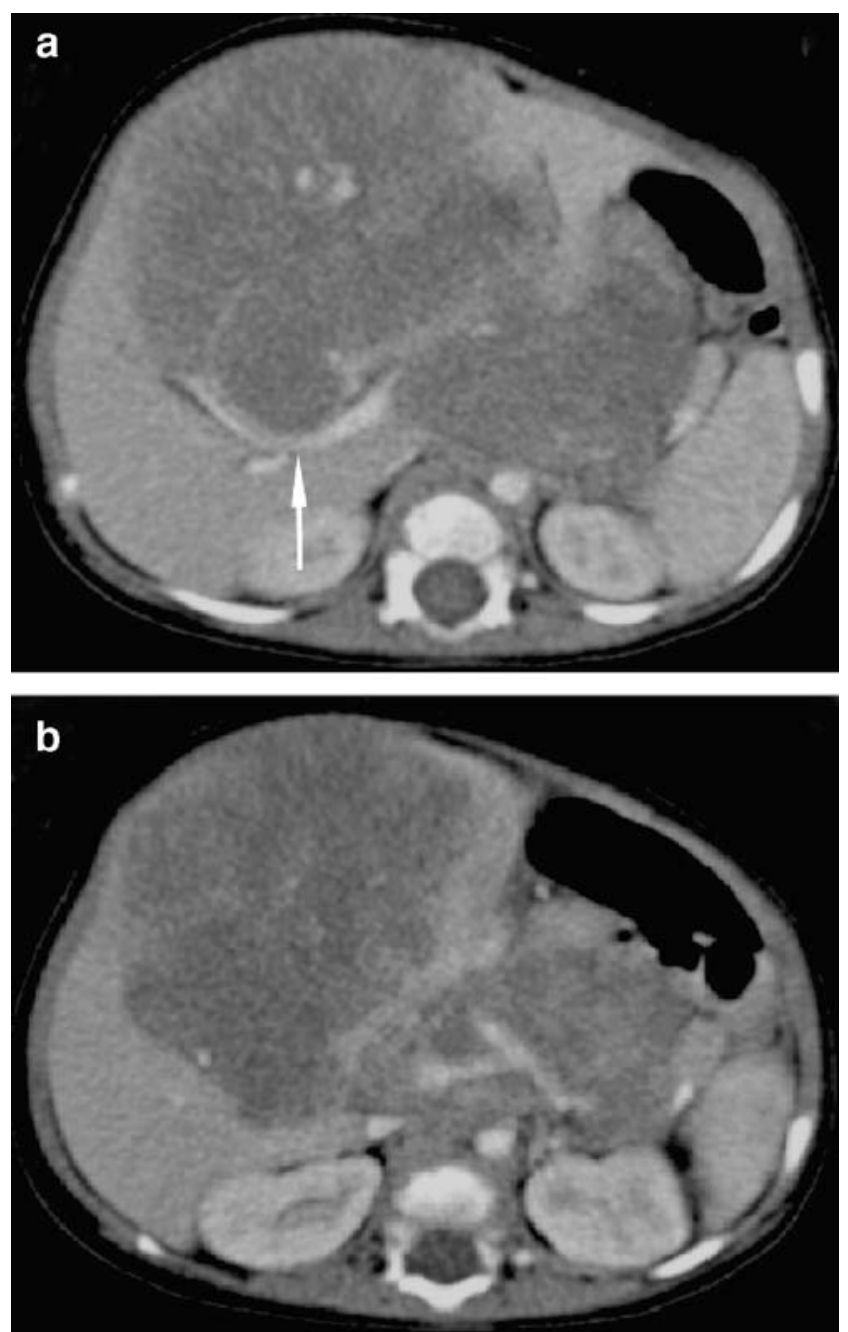

Fig. 3 Retroperitoneal extension in a patient with small-cell undifferentiated hepatoblastoma (E1). a CT shows a large PRETEXT III tumour with displacement of the right branch of the portal vein (arrow) and retroperitoneal extension (E1). b Image at a more caudal level shows involvement of the caudate lobe $(\mathrm{C} 1)$, encasement of the main portal vein (P2) and direct extension into the pancreas (E1)
(Fig. 3). When this occurs, the alternative diagnosis of pancreatoblastoma (another tumour that may secrete alphafetoprotein) with liver involvement should be considered.

Peritoneal spread of tumour is also unusual in children, but may be shown by cross-sectional imaging (Figs. 4 and 5). Ultrasonography is particularly sensitive in the detection of small peritoneal implants in the presence of ascites. It seems intuitively likely that this form of spread is prognostically worse than direct extension, and for this reason the 2005 revision defines peritoneal implants as E2. Biopsy proof is not required if the imaging findings are diagnostic.

In current SIOPEL protocols, children with hepatoblastoma and either E1 or E2 are assigned to the high-risk treatment group. This allocation is arbitrary, because there is so far no evidence from any large study that extrahepatic abdominal spread is an independent risk factor. All patients without evidence of E1 or E2 are coded as E0. Abdominal lymph node metastases are considered separately in the 2005 revision [3].

It is not known whether ascites, which is seen at presentation in a minority of children with primary liver tumours, is a marker of poor prognosis. The suffix " $\mathrm{a}$ " is added to the $\mathrm{E}$ number of patients with ascites (i.e. E0a, E1a or E2a as appropriate) in order to facilitate prospective analysis of its significance [3].

\section{Tumour rupture (intraperitoneal haemorrhage) at diagnosis (H1)}

Tumour rupture (Fig. 6) is a well-recognized presentation of both hepatoblastoma and hepatocellular carcinoma in childhood [8-10]. Its prognostic significance is not certain, but since the opening of the SIOPEL 4 study in September

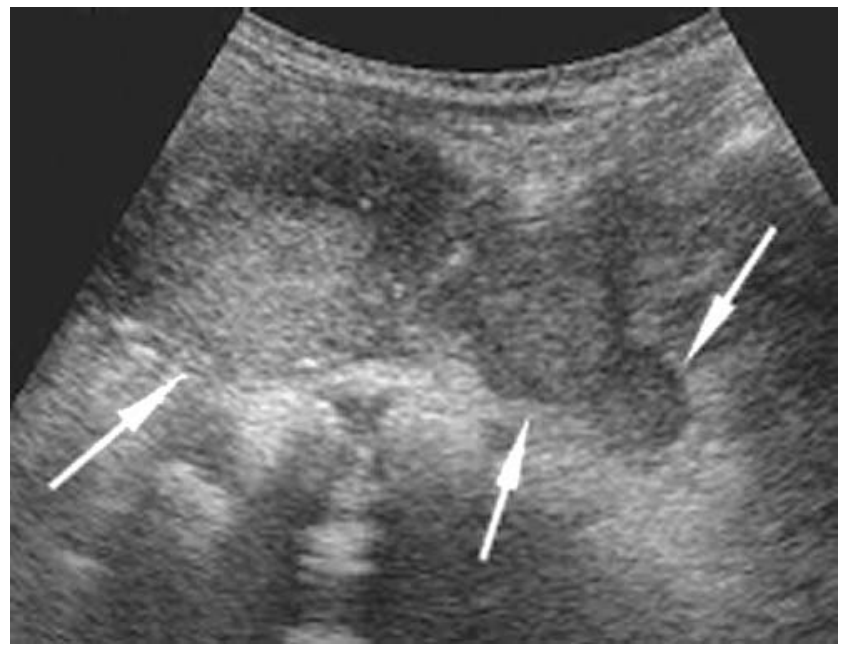

Fig. 4 Peritoneal tumour seeding in a child with hepatoblastoma (E2). US image shows peritoneal nodules (arrows). The diagnosis was confirmed by biopsy 

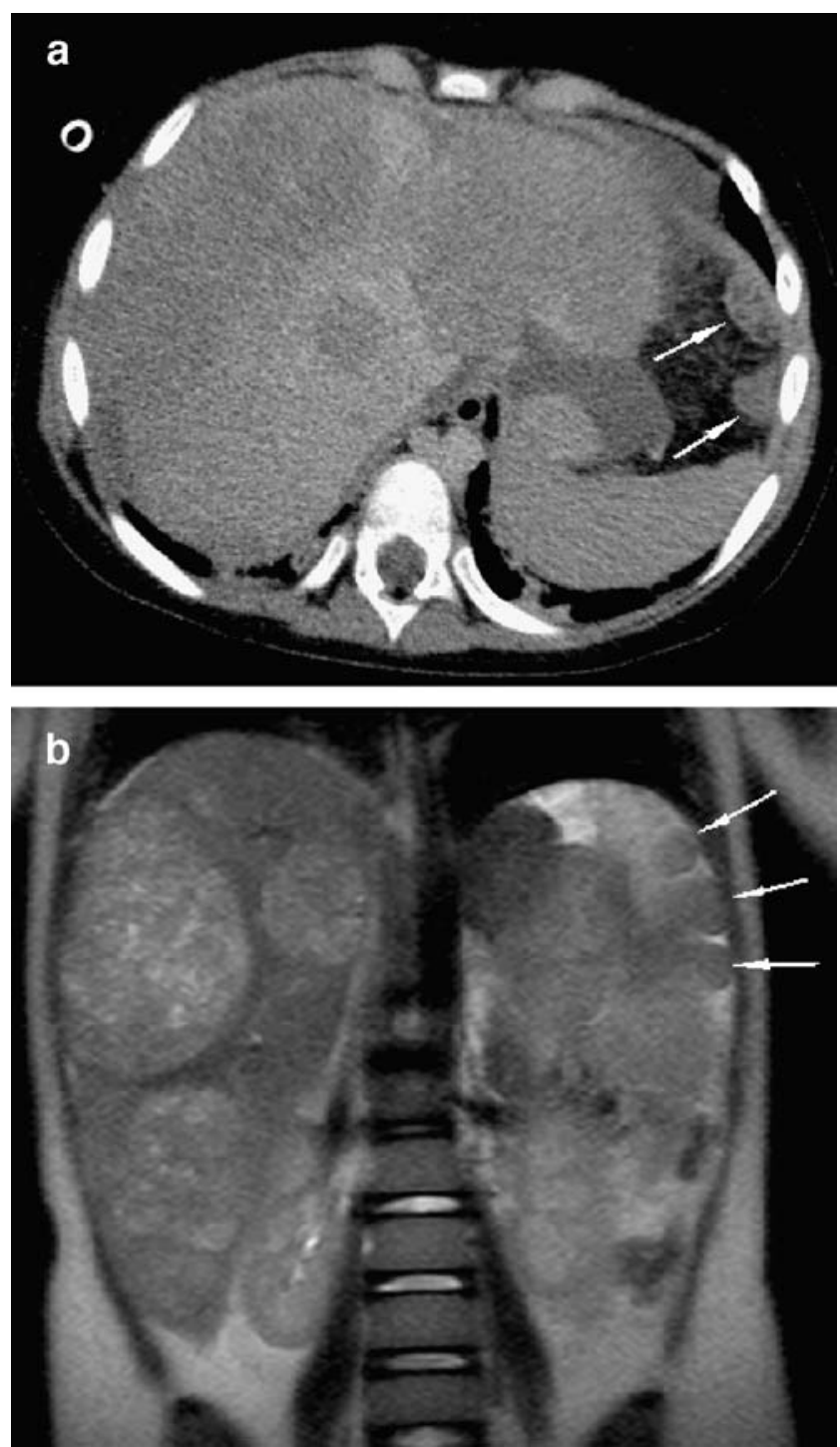

Fig. 5 Peritoneal tumour nodules and ascites in a child with multifocal hepatoblastoma (E2a). a CT shows peritoneal nodules (arrows). b Coronal MRI (HASTE sequence) shows nodules (arrows) with surrounding ascites

2004, tumour rupture (H1) has become a defining feature of high-risk hepatoblastoma in SIOPEL studies. It should be noted that characteristic imaging and clinical findings (such as hypotension and low haematocrit or haemoglobin level) are regarded as sufficient to make this diagnosis. Paracentesis is not recommended unless there is any clinical doubt. Embolization may be appropriate if there is continuing haemorrhage [11-13]. Localized or subcapsular haemorrhage (Fig. 7) is a fairly common finding and is specifically excluded from $\mathrm{H} 1$, as is biopsy-related intraperitoneal bleeding. Patients with no evidence of tumour rupture or haemorrhage are coded as $\mathrm{H} 0$ [3].

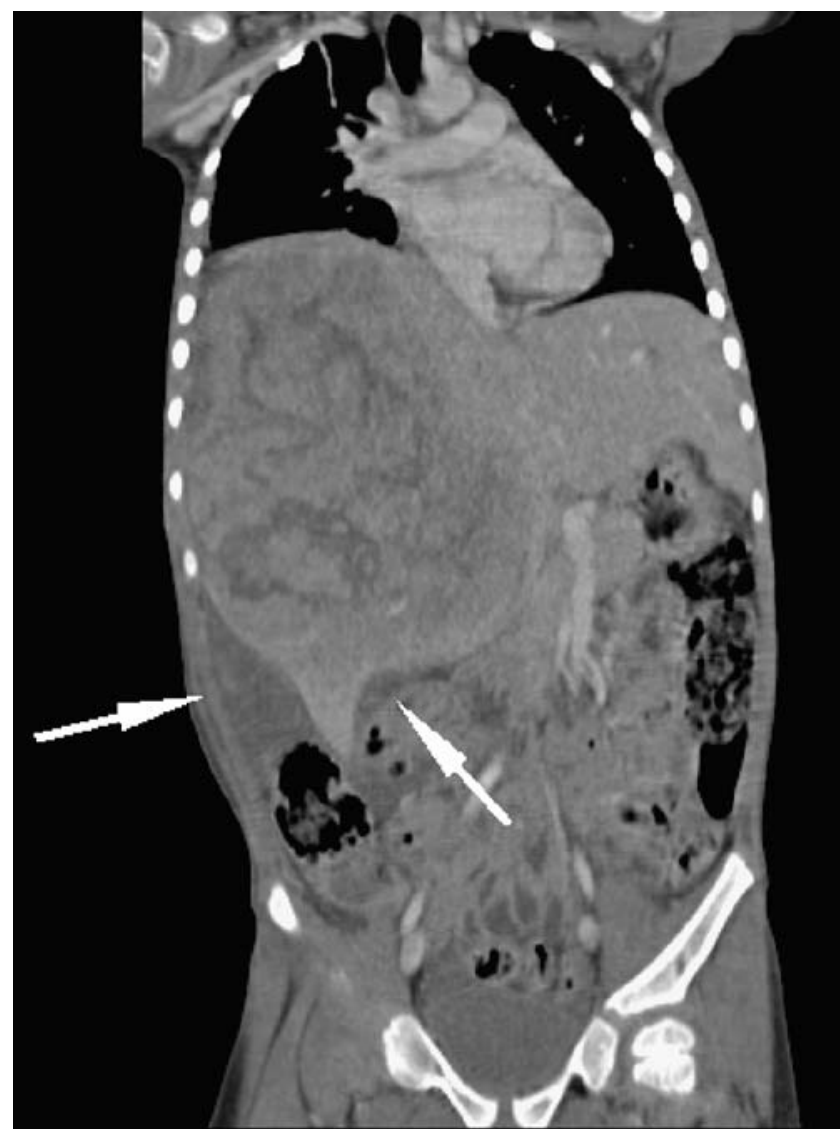

Fig. 6 Tumour rupture in a patient with newly diagnosed hepatoblastoma (H1). Coronal CT image shows high attenuation intraperitoneal fluid compatible with blood over the surface of the right lobe of the liver (arrows)

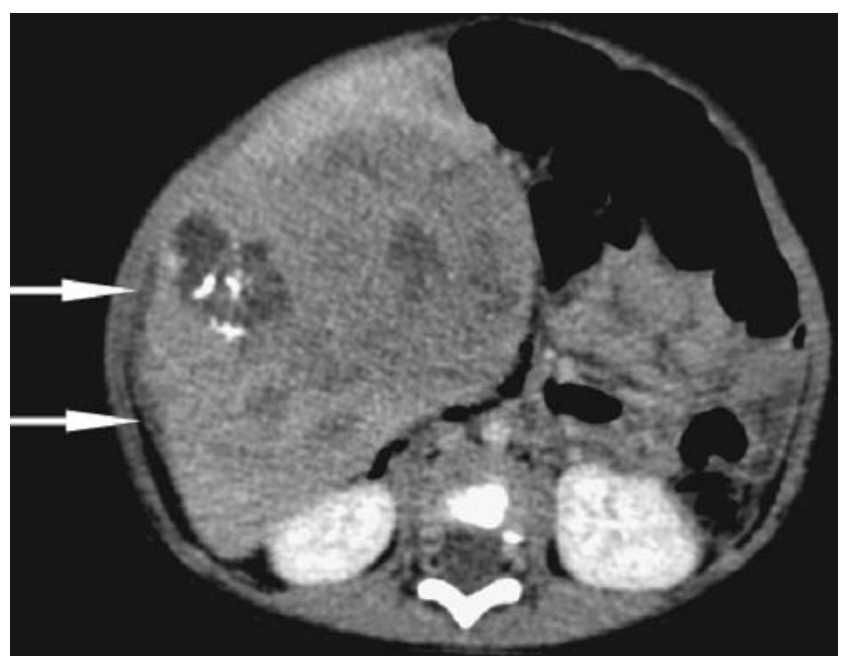

Fig. 7 Subcapsular haemorrhage in a patient with hepatoblastoma (H0). CT shows a thin crescent of fluid over the lateral surface of the right lobe (arrows). This is a common finding and does not affect staging 


\section{References}

1. Ortega JA, Douglass EC, Feusner JH et al (2000) Randomized comparison of cisplatin/vincristine/fluorouracil and cisplatin/continuous infusion doxorubicin for treatment of pediatric hepatoblastoma: A report from the Children's Cancer Group and the Pediatric Oncology Group. J Clin Oncol 18:2665-2675

2. Ortega JA, Krailo MD, Haas JE et al (1991) Effective treatment of unresectable or metastatic hepatoblastoma with cisplatin and continuous infusion doxorubicin chemotherapy: a report from the Children's Cancer Study Group. J Clin Oncol 9:2167-2176

3. Roebuck DJ, Aronson D, Clapuyt P et al (2007) 2005 PRETEXT: a revised staging system for primary malignant liver tumours of childhood developed by the SIOPEL group. Pediatr Radiol $37: 123-132$

4. Stocker JT, Schmidt D, Hamilton SR et al (2000) Hepatoblastoma. Pathology and genetics of tumours of the digestive system. IARC Press, Lyon, pp 184-189

5. Moore SW, Millar AJ, Hadley GP et al (2004) Hepatocellular carcinoma and liver tumors in South African children: a case for increased prevalence. Cancer 101:642-649
6. Roebuck DJ, Olsen O, Pariente D (2006) Radiological staging in children with hepatoblastoma. Pediatr Radiol 36:176-182

7. Lin MC, Wu CC, Chen JT et al (2005) Surgical results of hepatic resection for hepatocellular carcinoma with gross diaphragmatic invasion. Hepatogastroenterology 52:1497-1501

8. Chan KL, Fan ST, Tam PK et al (2002) Management of spontaneously ruptured hepatoblastoma in infancy. Med Pediatr Oncol 38:137-138

9. Iida T, Suenaga M, Takeuchi Y et al (2004) Successful resection of a ruptured hepatoblastoma prior to chemotherapy: report of a case. Surg Today 34:710-714

10. Ishak KG, Glunz PR (1967) Hepatoblastoma and hepatocarcinoma in infancy and childhood. Report of 47 cases. Cancer 20:396-422

11. Chan KL, Tam PK (1998) Successful right trisegmentectomy for ruptured hepatoblastoma with preoperative transcatheter arterial embolization. J Pediatr Surg 33:783-786

12. Lee SC, Chung JW, Kim KH et al (1999) Successful transumbilical embolization of congenitally ruptured hepatoblastoma. J Pediatr Surg 34:1851-1852

13. Ueno S, Hirakawa H, Yokoyama S et al (2005) Treatment of infantile hepatoblastoma and related complications. Tokai J Exp Clin Med 30:203-209 